\title{
BMJ Open Modified tissue-selecting therapy stapler combined with complete anal canal epithelial preservation operation for the treatment of circumferential mixed haemorrhoids: a protocol for single-blind randomised controlled study
}

\author{
Hua Huang, ${ }^{1}$ Yunfei Gu, ${ }^{2}$ Youran $\mathrm{Li}^{2}{ }^{2}$ Lijiang Ji (D) ${ }^{1}$
}

To cite: Huang H, Gu Y, Li Y, et al. Modified tissue-selecting therapy stapler combined with complete anal canal epithelial preservation operation for the treatment of circumferential mixed haemorrhoids: a protocol for single-blind randomised controlled

study. BMJ Open

2021;11:e052982. doi:10.1136/ bmjopen-2021-052982

- Prepublication history and additional supplemental material for this paper are available online. To view these files, please visit the journal online (http://dx.doi.org/10.1136/ bmjopen-2021-052982).

Received 11 May 2021 Accepted 30 October 2021

\section{Check for updates}

(c) Author(s) (or their employer(s)) 2021. Re-use permitted under CC BY-NC. No commercial re-use. See rights and permissions. Published by BMJ.

'Department of Anorectal, Changshu Hospital Affiliated to Nanjing University of Chinese Medicine, Changshu, Jiangsu, China

${ }^{2}$ Department of Anorectal, Affiliated Hospital of Nanjing University of Chinese Medicine, Nanjing, Jiangsu, China

Correspondence to

Lijiang Ji; JiL2235@outlook.com

\section{ABSTRACT}

Introduction This protocol designed a randomised controlled trial (RCT) to evaluate the effectiveness, safety and prognostic outcomes of modified tissue selecting technique (M-TST) combined with complete anal canal epithelial preservation (CACP) among patients with circumferential mixed haemorrhoids.

Methods and analysis This study will be single-blinded, and recruit 348 patients who are admitted to the Changshu Hospital Affiliated to Nanjing University of Chinese Medicine and fulfil the inclusion criteria from January 2022 to December 2022. Patients will be randomly assigned to the treatment group and the control group in a 1:1 ratio. The statistician will be blinded for the allocation. The treatment group will receive M-TST combined with CACP (M-TST-CACP), while the control group will receive the procedure for prolapse and haemorrhoids. The two groups will receive the same preoperative and postoperative care. The primary outcome will be recurrence rate. The secondary outcomes will be operation time, intraoperative bleeding, incontinence, pain, postoperative complications (severe bleeding, perianal oedema, urinary retention, faecal urgency, skin tags and anal stenosis), prolapse, recovery time, quality of life, Haemorrhoid Severity Score, and Symptom Severity Score.

Ethics and dissemination This protocol has been approved by the Clinical Ethics Committee of the Changshu Hospital Affiliated to Nanjing University of Chinese Medicine (approval no. 202102001). Trial registration number ChiCTR2100042750.

\section{INTRODUCTION}

Haemorrhoids are naturally presenting submucosal vascular tissue located in the anal canal, which tends to engorge and prolapse due to the ample vascular supply. ${ }^{12}$ Mixed haemorrhoids are a common type and appear both above and below the dentate line, and circumferential mixed haemorrhoid is the
Strengths and limitations of this study

This is a randomised controlled trial to evaluate the effectiveness, safety and prognostic outcomes of a novel treatment method (modified tissue selecting technique combined with complete anal canal epithelial preservation operation) for circumferential mixed haemorrhoids.

- All surgeons involved are experienced in the new treatment method and trained by the same specialist.

- This is a single-centre trial, which is a limitation of this study.

- Due to the limitations of the treatment, it is difficult for surgeons, patients and evaluators to blind.

- The applicability of this treatment in patients with internal haemorrhoids at grade II remains unclear because our study only includes those at grade III or IV.

final stage of haemorrhoidal development. ${ }^{3}$ The most widely recommended treatment option of which, according to the American Society of Colon and Rectal Surgeons Clinical Practice Guidelines, is surgical haemorrhoidectomy. ${ }^{4}$

The mainstream surgical treatments are the open excisional (Milligan-Morgan) haemorrhoidectomy (MMH), closed (Ferguson) haemorrhoidectomy, submucosal haemorrhoidectomy, stapled haemorrhoidectomy and laser haemorrhoidectomy. In the west, transanal haemorrhoidal dearterialisation (THD) and rubber band ligation (RBL) are also common treatment options. ${ }^{56}$ Currently, no surgical treatment has been recommended as a gold standard for circumferential mixed haemorrhoids. MMH is the classical surgery 
for mixed haemorrhoids, but it often causes anal stenosis, incontinence and obvious pain in circumferential mixed haemorrhoid, affecting patients' daily life. ${ }^{7}$ As a substitute for $\mathrm{MMH}$, the procedure for prolapse and haemorrhoids (PPH) is proposed. ${ }^{8}$ The PPH resects the prolapsed rectal mucosa on the dentate line using a stapler, which protects the anal canal, posing less damage to the anal epithelium and dentate line. ${ }^{9}$ However, the PPH is associated with a significantly higher recurrence rate than the $\mathrm{MMH}$, varying from $3.4 \%$ to $45 \%$, and usually appears residual external haemorrhoids. ${ }^{10-12}$ Therefore, surgeons domestic and abroad devote to explore an optimal treatment to protect anal function and decrease the recurrence rate.

Tissue selecting technique (TST) is a surgical method based on the PPH and combined with the theory of 'segmented dentate ligation' in traditional Chinese medicine. ${ }^{13}$ TST can depend on the number and size of haemorrhoid nucleus to select the scope of haemorrhoid mucosa resection, and realise the modern minimally invasive concept. ${ }^{13} \mathrm{~A}$ meta-analysis showed that the recurrence rate of TST was the lowest when compared with the PPH and MMH. ${ }^{13}$ However, TST inherits the defect of PPH that pathological anal cushion is not handled and the 'suspension' effect of TST is inferior to that of PPH. ${ }^{14}$ To improve this, we move down the purse position, which can selectively resect 1/3-2/3 pathological anal cushion while improving the 'suspension' effect. In addition, for mild internal haemorrhoids, hardener is injected to make submucosal tissues produce extensive fibrosis and adhesion, which can both reduce haemorrhoids blood supply to accelerate the atrophy of haemorrhoids, and also play a role in the upward suspension and traction of prolapsed anal cushion. This we called is modified TST (M-TST). The clinical study has showed that M-TST is superior to $\mathrm{MMH}$ in the operation time, postoperative pain and recovery time. ${ }^{14}$

As the awareness of anal function protection increases in recent years, anorectal surgeons are no longer limited to the protection for anal cushion on the dentate line, but also for the protection of canal epithelial under the dentate line, excessive coloboma of which can cause scar hyperplasia, resulting in anal stenosis and anal dysfunction. ${ }^{15} \mathrm{Gu}$ et al have developed complete anal canal epithelial preservation (CACP) operation that perform an arc-incision on the external haemorrhoids to preserve the epithelium of the anal canal, which mainly applied for the treatment of external haemorrhoids in grade III or IV mixed haemorrhoids. ${ }^{16}$ The combination of M-TST and CACP (M-TST-CACP) can protect the anal canal epithelium while ensuring the resection of the lesion tissue. A recent study shows that the M-TST-CACP significantly decreases the anal pain and has advantages in protecting anal function and improving patients' satisfaction compared with $\mathrm{MMH},{ }^{17}$ indicating the clinical application value.

This protocol attempted to design a randomised controlled trial (RCT) with a larger study sample to compare the efficacy and safety of M-TST-CACP with PPH.

\section{METHODS AND ANALYSIS}

\section{Trial design and setting}

This prospective single-blinded randomised control study will be conducted in a single-centre, at Changshu Hospital Affiliated to Nanjing University of Chinese Medicine. Participants who fulfil the inclusion criteria will be recruited from January 2022 to December 2022 at Changshu Hospital Affiliated to Nanjing University of Chinese Medicine. Patients will be randomised into two groups in a 1:1 ratio, with each group containing 174 participants. The treatment group will receive M-TSTCACP, while the control group will receive PPH. The overall duration of the study will be 24 months. The study period of this research will be 12 months. The timeline of the study period is summarised in table 1 . The overall process of the study protocol was presented in figure 1 .

\section{Eligibility criteria}

Inclusion criteria

1. Aged 18-70 years.

2. Diagnosed with circumferential mixed haemorrhoids with internal haemorrhoids at grade III or IV. ${ }^{18}$

3. No anal morphological and functional abnormalities before the surgical operation.

4. Agreeing and signing the informed consent voluntarily.

\section{Exclusion criteria}

1. With history of haemorrhoids or other anorectal surgery.

2. Pregnant, breastfeeding, or experiencing a menstrual period.

3. Acute inflammatory or thrombosed external haemorrhoids.

4. Rectal cancer, rectal polyps, tuberculosis, Crohn's disease, or other rectal and anal diseases.

5. Severe raspatory, digestive, renal, hepatic, circular system, and blood system health conditions.

6. Participating or had participated in other clinical studies within 30 days.

\section{Blinding and allocation}

A sequential number, from 0 to 348 , will be generated and assigned to each participant by computer. After recruitment, the concealed allocation will be performed by a computer-generated randomisation list. The sequential number will be placed in sealed opaque envelopes and kept by trained professionals. The statistician will be blinded for the allocation of the study participants. Patients in the treatment and control group will receive equivalent preoperative and postoperative care.

\section{Potential bias}

Potential bias will be addressed mainly by the study design and prestudy training: (i) The single-blinded randomised control design will diminish the potential selection bias, 
Table 1 Timeline of the study

\begin{tabular}{|c|c|c|c|c|c|c|c|}
\hline \multirow[b]{2}{*}{$\begin{array}{l}\text { Time point } \\
\text { Projects }\end{array}$} & \multicolumn{2}{|c|}{ Preliminary phase } & \multicolumn{3}{|c|}{ Hospitalisation } & \multicolumn{2}{|l|}{ Follow-up } \\
\hline & $\begin{array}{l}\text { January 2022- } \\
\text { December } 2022\end{array}$ & $\begin{array}{l}\text { 0-3 days after } \\
\text { admission }\end{array}$ & $\begin{array}{l}1 \text { day after } \\
\text { surgery }\end{array}$ & $\begin{array}{l}7 \text { days after } \\
\text { surgery }\end{array}$ & Discharge & $\begin{array}{l}1 \text { month } \pm 7 \\
\text { days }\end{array}$ & $\begin{array}{l}12 \\
\text { months } \pm 30 \\
\text { days }\end{array}$ \\
\hline Recruitment & $x$ & & & & & & \\
\hline Baseline examination & & $x$ & & & & & \\
\hline Medical history & & $x$ & & & & & \\
\hline Physical examination & & $x$ & & & & & \\
\hline Eligibility & & $x$ & & & & & \\
\hline Informed consent & & $x$ & & & & & \\
\hline Operation time & & & $x$ & & & & \\
\hline Intraoperative bleeding & & & $x$ & & & & \\
\hline Pain & & & $x$ & $x$ & & & \\
\hline Postoperative complications & & & & $x$ & & & $x$ \\
\hline Incontinence & & & & & & $x$ & $x$ \\
\hline Recovery time & & & & & & $x$ & \\
\hline Prolapse & & & & & & & $x$ \\
\hline Recurrence rate & & & & & & & $\mathrm{X}$ \\
\hline Quality of life & & & & & & & $x$ \\
\hline Haemorrhoid severity score & & & & & & $x$ & $x$ \\
\hline Symptom severity score & & $x$ & & & & & $x$ \\
\hline Medications use & & & & & $x$ & $x$ & $x$ \\
\hline Data analysis and research conclusion & & & & & & & $x$ \\
\hline
\end{tabular}

reduce the influence of potential covariates and increase the validity of the results. (ii) Prior to the beginning of the study, all researchers and office staff will be trained and informed according to the research protocol. Being

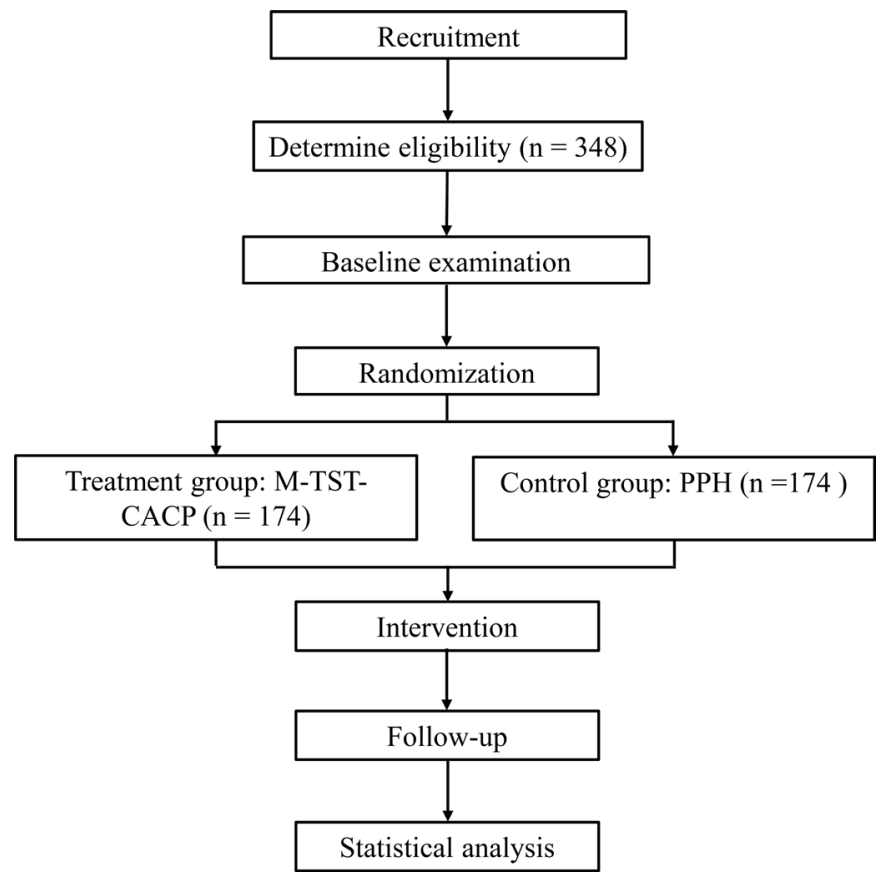

Figure 1 Flow chart of the study protocol. M-TST-CACP, modified tissue selecting technique combined with complete anal canal epithelial preservation; $\mathrm{PPH}$, procedure for prolapse and haemorrhoids. familiar with the study protocol will decrease the potential bias during the data collection and processing stage.

\section{Clinical interventions}

All patients will be required to fast for at least 12 hours before surgery. Routine blood sample screening, urinary sample, hepatic function, renal function and infectious disease indicators will be conducted. ECG, chest X-ray, abdominal ultrasound, colonoscopy, pathological analysis through biopsy will also be performed to determine the eligibility. All operations will be performed under spinal anaesthesia for all participants. All patients will be placed in the left lateral decubitus position during the operation. All surgeons involved are experienced, with experience more than 15 years to treat anorectal diseases. The $33 \mathrm{~mm}$ disposable open-loop minimally invasive anorectal stapler (Touchstone International Medical Science Co, Ltd, Suzhou, China) will be used for PPH and M-TST.

\section{Treatment group: M-TST-CACP}

Single-opening, double-opening or triple-opening anoscopes will be chosen based on the number, size and location. After fully dilatating the anus, paraffin oil-lubricated anoscope will be inserted into the anus, and adjusted until mucosa over the haemorrhoids to be resected is fully exposed to the window. After fixing the anoscope, segmented purse-string suture is performed on the mucosa and submucosa using a 7-gauge silk thread at $2 \mathrm{~cm}$ above the dentate line. When the stapler opens to its maximum position, the stapler head will be placed 

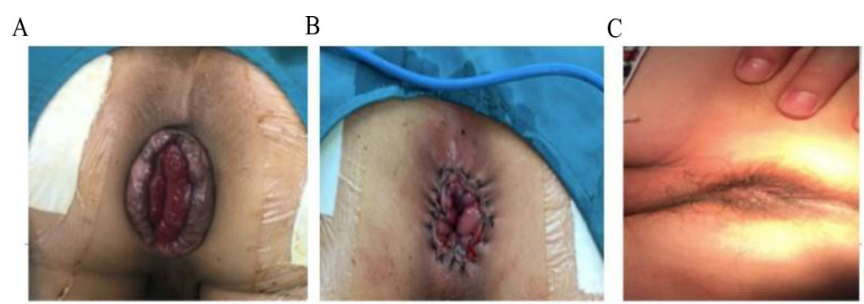

Figure 2 Pictures for CACP before operation (A), after operation (B) and 3 months after the operation (C). CACP, complete anal canal epithelial preservation.

above the purse-string suture, go through the purse-string suture and pull the purse-string suture to tie. The traction is continuous to make the mucosa into the stapler groove. The stapler will be tightened and fired. Waiting for $30 \mathrm{~s}$, the stapler is loosened and stapler head is taken out. After sufficient haemostasis, the anoscope will be removed. For mild internal haemorrhoids, $5 \mathrm{~mL}$ lauromacrogol (Shanxi Tianyu Pharmaceutical Co., Ltd., Chinese medicine standard word H20080445, specifications: $10 \mathrm{~mL}: 100 \mathrm{mg}$ ) will be used for high and low injection of submucosa at relaxation place of mucosa. Then, two sides of the external haemorrhoids will be clamped using vascular clamp, and arc-shaped incision will be made by the dermatome in the anal canal skin line. Figure 2 illustrates the CACP. If severe varicose veins are present, the incision undermining dissection will be performed, and the skin edge will be trimmed until it is smooth. Mattress suture will be performed using 3-0 absorbable thread for the skeleton of the incision, while 4-0 absorbable thread will be used for both ends and the suture gap. Pressure bandages will be applied after ensuring haemostasis. The resected tissue will undergo a pathological examination.

\section{Control group: PPH}

After spinal anaesthesia and routine disinfection, routine anal enlargement is performed, and 7-gauge silk thread is used to suture anal edge at 8 o'clock, 4 o'clock and 12 o'clock directions. The suture will be pulled to emerge the mucous and haemorrhoids. The anal dilator and anoscope are relatively placed and fixed. The $2-0$ absorbable thread is used to annularly suture the mucous. The purse-string suture is $3 \mathrm{~cm}$ above the dentate line. The stapler will be placed above the suture. The suture will be tightened and tied, and threading apparatus is applied to lead the suture out of the anus. The suture traction and pull operation is performed to make the stapler casing enter the rectal mucosa. The stapler will be tightened to allow resection. After resection, the surgeon will remove the stapler and examine it for any bleeding. Additional absorbable sutures will be performed if bleeding occurs. Patients with residual external haemorrhoid will be added with haemorrhoidectomy. Vaseline will be applied to the wounded area.

\section{Postoperative care}

All patients will receive intravenous antibiotics to prevent infection. Additionally, patients will be administered a liquid diet 2 hours after surgery and return to regular diet 24 hours after surgery. After the first defecation, patients will be allowed to bathe. The bandage will be changed in the morning, at night, and after defecation.

\section{Prognostic outcomes}

Baseline characteristics, and prognostic outcomes will be collected and compared between the treatment and control groups. Baseline characteristics will contain age, sex, ethnicity, height, weight and lifestyle will be collected. The medical history of the patients will also be collected.

\section{Primary outcomes}

The primary prognostic outcome is the recurrence rate, which will be evaluated by inpatient visits and phone call follow-ups at 12 months. According to Shanmugam et $a l,{ }^{19}$ the outcome is binary. Patients are considered cured or improved if they are asymptomatic or had minimal residual symptoms that not require further treatment at the end of the study period. Patients are considered unchanged or worse if symptoms are not improved, or deteriorated for further intervention or surgical complications appear. Based on this, the following conditions are considered to be recurrent: (1) patient reports 'unchanged or worse compared with before starting treatment' at 12 months; (2) any subsequent procedures, such as RBL, haemorrhoidal artery ligation, THD, haemorrhoidectomy, haemorrhoidal injection or other related procedures, in the past 12 months and (3) any symptoms or events highly indicated recurrent haemorrhoids are presented (adjudicated by the investigators).

\section{Secondary outcomes}

Intraoperative outcomes

1. Operation time: the interval starting from the initiation of the operation to the application of the dressing.

2. Intraoperative bleeding: the quantified blood loss will be evaluated based on the use of medical gauze. Each soaked gauze equals $5 \mathrm{~mL}$ blood loss.

\section{Postoperative outcomes}

1. Incontinence: anal incontinence will be evaluated at 1 and 12 months of the follow-up using the Wexner Faecal Incontinence Score, ${ }^{20}$ as presented in table 2. The scale scores from 0 to 20, and higher score indicates more serious degree of incontinence.

2. Pain: the visual analogue scale will be used to evaluate pain. Patients will be presented a $10 \mathrm{~cm}$ horizontal line with 0 (no pain at all) to 10 (worst pain imaginable). The pain will be assessed at 1 day and 7 days after surgery.

3. Postoperative complications

The following complications are assessed at 7 days after surgery.

a. Severe bleeding: the bleeding necessitating prolonged hospital stay, readmission or transfusion.

b. Perianal edema: Perianal edema will be graded from I to IV. Grade I represent no edema. Grade II indicates mild edema, affecting $25 \%$ of the anal area, which does 
Table 2 The Wexner score assessing anal incontinence

\begin{tabular}{llllll}
\hline & Frequency & & & \\
\cline { 2 - 6 } Types of incontinence & Never & Rarely & Sometimes & Usually & Always \\
\hline Solid & 0 & 1 & 2 & 3 & 3 \\
Liquid & 0 & 1 & 2 & 3 & 4 \\
Gas & 0 & 1 & 2 & 3 & 3 \\
Wear pad & 0 & 1 & 2 & 4 \\
Lifestyle altered & 0 & 1 & 2 & 4 \\
\hline
\end{tabular}

Frequency definition: never-0, rarely-less than once a month, sometimes-less than once a week or once a month, usually-once a day or once a week, always-once a day or more. Score: perfect anal continence-0, complete anal incontinence-20.

not impact any activity. Patients who experience moderate edema, occupying $50 \%$ of the anus and restricted activity are classified as Grade III. Grade IV refers to severe edema that impacts daily activities.

c. Urinary retention: patients who need urinary catheterisation are considered urinary retention.

d. Fecal urgency: patients will be asked to answer 'yes' or 'no' for the occurrence of fecal urgency.

The following complications are assessed at 12 months after surgery.

e. Skin tags: anal skin tags are divided into four grades. ${ }^{21}$ Grade I refers to smooth and flat anal areas with no skin tags. Patients with less than three local areas that are slightly convex and asymptomatic are grade II. Patients who were prominently raised and asymptomatic were evaluated as grade III. Patients with more than three locally raised bumps and asymptomatic were evaluated as class IV.

f. Anal stenosis: anal stenosis is identified when the normal elastic anal canal becomes abnormally tight and fibrous. ${ }^{22}$

4. Postoperative defecation: during hospitalisation, the first defecation after surgery will be recorded.

5. Length of the hospital stay.

6. Recovery time: the interval from the end of the operation to the recovery, which is defined as patients are asymptomatic or had minimal residual symptoms that not require further treatment. ${ }^{19}$ The recovery time will be evaluated at 1 month in the period of follow-up.

7. Prolapse: assessed at 12 months after the surgery.

8. Quality of life: the patients' health status will be evaluated using the EuroQol-5D-3L descriptive system score at 12 months follow-ups. ${ }^{23}$

9. Haemorrhoid severity score: this score is used to compare the severity of haemorrhoidal symptoms, and the difference in symptom severity is relatively compared for the 1 month and 1 year time points. ${ }^{6}$

10. Symptom severity score: this score contains five questions and the total score is 15 . The increase in score means the increase in symptoms. ${ }^{25}$ This questionnaire is submitted before the surgery and at 12 months of follow-up.

\section{Recruitment}

Patients will be recruited by the recruitment team, consisting of coloproctologists and trained medical professionals. Patients who are admitted to the inpatient department at Changshu Hospital and fulfil the enrolment eligibility will be recruited. The recruitment team will be trained prior to the research to ensure the quality of the recruitment.

\section{Study completion and discontinuation}

\section{Study completion}

Patient has completed the entire 12 months study period, including the treatment and the follow-up visits.

\section{Study discontinuation}

The study will be discontinued under the following conditions:

1. Patient withdraws informed consent and requires to quit voluntarily.

2. Pregnancy.

3. Any situation will lead a potential safety risk to the patient in the trial.

\section{Withdrawal}

All patients will be participating in the study voluntarily and have the right to withdraw consent from the study for any reason at any time. When the patient has withdrawn the consent to participate, the study must be discontinued, and the collected data will be considered missing. The following situation will be considered withdrawing from the study:

1. Refuse to continue the study

2. Refuse any further visits or assessment

3. Refuse any further study-related contacts

4. Refuse any use of collected data by the researcher

\section{Data collection and management}

Data collection

After a thorough literature review and discussion with the expert in the field, the research will design a Case Report Form to collecting study-related information. Data will be collected and entered into the Electronic Data Capture (EDC) system by designated investigators. Collected data will be handled by data management staff. 


\section{Quality control}

All staff will participate in special training to know about the aim and content of the study, treatment methods and quality control before the study. Monitors will visit the site to check the completeness of patient records, the accuracy of data capture and data entry, the adherence to the protocol and the progress of enrolment to ensure the integrity and quality of the data during the study implementation period. Dropouts and withdrawals including the reasons will be recorded in detail through the trial. The information about patients will be placed in locked file cabinets at the study site with limited access. Only investigators have right to access the information, and all of them will always strictly maintain a privacy policy to protect confidentiality before, during and after the trial.

\section{Statistical analysis}

The normally distributed continuous variables will be presented as mean \pm SD. The intergroup comparisons of normally distributed continuous variables will be performed by the independent sample t-test, while the intragroup comparison will be conducted using paired sample t-test. Non-normally distributed variables will be expressed in median, and compared by the Mann-Whitney $\mathrm{U}$ test. The count variables will be compared using the Pearson $\chi^{2}$ test or the Fisher's exact test, and expressed as number $(\mathrm{n})$ and percentage $(\%) . \mathrm{p}<0.05$ is regarded as statistical significance. In addition, all statistical analyses will be two-tail tests. SPSS V.24.0 (SPSS, Chicago, IL, USA) software will be used for the statistical analysis.

\section{Sample size}

The hypothetical 12-month recurrence rate of the control group will be determined as $10 \%,{ }^{26}$ and the hypothetical rate of the treatment group will be set as $2 \%$ according to the past cases in our hospital. Assuming $\alpha=0.05$ and $\beta=0.15$ of the two-tailed test, the sufficient sample size of each group will be 157 cases. Furthermore, considering the potential missing data and hypothetical $10 \%$ dropout rate, the sufficient sample size of each group will be 174 cases. Therefore, the final total sample size will be 348 cases.

\section{Patient and public involvement}

In the absence of a patient association, we form a panel of three patients with circumferential mixed haemorrhoids at grade III or IV to think along and comment on our protocol design. This panel analysed the willingness to participate, patient information sheet, burden for patient, but they are not involved in the recruitment of the study. To improve the study, we evaluate patient burden with the panel jointly. The study results present to all participants via a seminar after termination of the study. The authors also thank the involved panel.

\section{Ethics and dissemination}

This protocol has been approved by the Clinical Ethics Committee of the Changshu Hospital Affiliated to the Nanjing University of Chinese Medicine (approval no.
202102001). All patients will receive the information about the study in verbal or written forms, and will sign the informed consent before the study. The research will be carried out in accordance with the Declaration of Helsinki. The study team will devote to disclosing results of the study. The results of the study will be disseminated as articles published in international peer-reviewed journals. The study will be performed and reported based on the consolidated standards of reporting trials statement. Prior to submission, the appropriateness and scientific merit of the paper will be reviewed by the appropriate subcommittee. The study team will follow the authorship criteria defined by the International Committee of Medical Journal Editors.

\section{Informed consent}

Investigators will disclose the background, objectives, protocol and risks to the patients in detail and answer all research-related questions proposed by the patients. The informed consent form (online supplemental file 1) must be signed voluntarily by the patients after complete disclosure of the research.

\section{Adverse event}

An adverse event is any untoward medical occurrence in a participant. Since the informed consent form being signed until the end of the study, any adverse event must be recorded and entered in the EDC adverse event form, the information of which should include the date, duration, severity, symptoms or characteristics, laboratory examinations, recurrence if any and clinical significance. Investigators must evaluate the adverse event and apply the appropriate treatment. The adverse event must be reported to the institutional review board for ethnic review.

\section{DISCUSSION}

This study protocol is designated to investigate the efficacy and safety of M-TST-CACP. Our previous study had compared the effectiveness of M-TST-CACP with MMH, which revealed promising results in pain decrease, anal function protection and improvements in patients' satisfaction. ${ }^{14}$ Nevertheless, the final sample size of this study was limited, with 80 cases in total. Additionally, the follow-up period was only 6 months. Thus, this protocol designed a study with a longer duration of the follow-up, 12 months and a larger sample size, 348 cases.

$\mathrm{MMH}$ is a conventional surgical treatment for mixed haemorrhoids, but it is commonly accompanied by adverse postoperative prognostic outcomes, such as anal incontinence, anal stenosis and severe pain, when implemented on patients with circumferential mixed haemorrhoids. ${ }^{4} 7$ In 1998, Longa et al proposed PPH as an effective alternative for grades II-IV circumferential mixed haemorrhoids, which can better preserve the anal tissue and function. ${ }^{8}$ The effectiveness, safety, pain and postoperative complication of the PPH have been 
evaluated in previous studies, the result of which showed promising outcomes compared with MMH. ${ }^{12} 2829$ Therefore, comparing M-TST-CACP with PPH will better evaluate the effectiveness and prognosis, and generate more meaningful clinical implications.

This is a RCT to evaluate the effectiveness, safety and prognostic outcomes of M-TST-CACP for circumferential mixed haemorrhoids. The surgery is performed by surgeons all experienced in the new method and trained by the same specialist. The single-centre design will be one limitation of the study. All participants will be recruited at Nanjing, Jiangsu province, China, the findings of which may require careful generalisation on populations with distinctive characteristics. Future multicentre studies may include populations with more diverse races and cultural backgrounds. In addition, its applicability in patients with internal haemorrhoids at grade II will be also explored in the future.

Contributors HH and LJ designed the study. HH wrote the manuscript. YG and YL collected, analysed and interpreted the data. LJ critically reviewed, edited and approved the manuscript. All authors read and approved the final manuscript.

Funding This study was supported by Changshu Municipal Science and Technology Bureau Supporting Project (CS201925), Special Project of Diagnosis and Treatment Technology for Key Clinical Diseases in Suzhou (LCZX202022) and Science and technology project of Changshu Health Committee (cswsq202007).

Competing interests None declared.

Patient consent for publication Consent obtained directly from patient(s).

Provenance and peer review Not commissioned; externally peer reviewed.

Supplemental material This content has been supplied by the author(s). It has not been vetted by BMJ Publishing Group Limited (BMJ) and may not have been peer-reviewed. Any opinions or recommendations discussed are solely those of the author(s) and are not endorsed by BMJ. BMJ disclaims all liability and responsibility arising from any reliance placed on the content. Where the content includes any translated material, BMJ does not warrant the accuracy and reliability of the translations (including but not limited to local regulations, clinical guidelines, terminology, drug names and drug dosages), and is not responsible for any error and/or omissions arising from translation and adaptation or otherwise.

Open access This is an open access article distributed in accordance with the Creative Commons Attribution Non Commercial (CC BY-NC 4.0) license, which permits others to distribute, remix, adapt, build upon this work non-commercially, and license their derivative works on different terms, provided the original work is properly cited, appropriate credit is given, any changes made indicated, and the use is non-commercial. See: http://creativecommons.org/licenses/by-nc/4.0/.

ORCID iD

Lijiang Ji http://orcid.org/0000-0001-9402-2451

\section{REFERENCES}

1 Lawrence A, McLaren ER. External Hemorrhoid. StatPearls Publishing, 2020. https://www.ncbi.nlm.nih.gov/books/NBK500009/

2 Fontem RF, Eyvazzadeh D. Internal Hemorrhoid. StatPearls Publishing, 2020. https://www.ncbi.nlm.nih.gov/books/NBK537182/

3 Lu B, Du J, Wu X. The effects of modified Buzhong Yiqi decoction combined with Gangtai ointment on the wound healing and anal function in circumferential mixed hemorrhoid patients. Am J Transl Res 2021;13:8294-301.

4 Davis BR, Lee-Kong SA, Migaly J, et al. The American Society of colon and rectal surgeons clinical practice guidelines for the management of hemorrhoids. Dis Colon Rectum 2018;61:284-92.

5 Trenti L, Biondo S, Kreisler Moreno E, et al. Short-Term outcomes of transanal hemorrhoidal dearterialization with Mucopexy versus vessel-sealing device hemorrhoidectomy for grade III to IV hemorrhoids: a prospective randomized multicenter trial. Dis Colon Rectum 2019;62:988-96.

6 Brown S, Tiernan J, Biggs K, et al. The HubBLe trial: haemorrhoidal artery ligation (HAL) versus rubber band ligation (RBL) for symptomatic second- and third-degree haemorrhoids: a multicentre randomised controlled trial and health-economic evaluation. Health Technol Assess 2016;20:1-150.

7 Bhatti MI, Sajid MS, Baig MK. Milligan-Morgan (open) versus Ferguson haemorrhoidectomy (closed): a systematic review and meta-analysis of published randomized, controlled trials. World $J$ Surg 2016;40:1509-19.

8 Longo A. 6th world congress of endoscopic surgery (IFSES)6th international congress of the european association of endoscopic surgery (E. A.E.S.) 1st world expo of surgical TechnologiesRome, italy, 31 may-6 june 1998 Oral presentations. Surg Endosc 1998;12:475-658.

9 Wang G-qiang, Liu Y, Liu Q, et al. [A meta-analysis on short and long term efficacy and safety of procedure for prolapse and hemorrhoids]. Zhonghua Wai Ke Za Zhi 2013;51:1034-8.

10 Oughriss M, Yver R, Faucheron J-L. Complications of stapled hemorrhoidectomy: a French multicentric study. Gastroenterol Clin Biol 2005;29:429-33.

11 You YN, Hardiman KM, Bafford A, et al. The American Society of colon and rectal surgeons clinical practice guidelines for the management of rectal cancer. Dis Colon Rectum 2020;63:1191-222.

12 Yang J, Cui P-J, Han H-Z, et al. Meta-Analysis of stapled hemorrhoidopexy vs Ligasure hemorrhoidectomy. World J Gastroenterol 2013;19:4799-807.

13 Zhang G, Liang R, Wang J, et al. Network meta-analysis of randomized controlled trials comparing the procedure for prolapse and hemorrhoids, Milligan-Morgan hemorrhoidectomy and tissueselecting therapy stapler in the treatment of grade III and IV internal hemorrhoids(Meta-analysis). Int J Surg 2020;74:53-60.

14 Sun Y, YF G. Clinical comparison between modified TST and Milligan-Morgan in the treatment of mixed hemorrhoids. Contemporary Medicine 2015;21:75-7.

15 Ji L, Li L, Weng L, et al. Tissue selecting technique mega-window stapler combined with anal canal epithelial preservation operation for the treatment of severe prolapsed hemorrhoids: a study protocol for a randomized controlled trial. Medicine 2020;99:e23122.

16 Huang $\mathrm{H}, \mathrm{Li}$ Y, Lu Y. The progress in complete anal canal preservation. J Colorectal Anal Surg 2019;25:481-3.

17 Huang H, YR L, YF G. Clinical observation of modified tissue selecting technique combined with complete anal canal epithelial preservation operation in the treatment of circumferential mixed hemorrhoids. Jie Zhi Chang Gang Men Wai Ke 2020;26:713-7.

18 Mott T, Latimer K, Edwards C. Hemorrhoids: diagnosis and treatment options. Am Fam Physician 2018;97:172-9.

19 Shanmugam V, Thaha MA, Rabindranath KS, et al. Systematic review of randomized trials comparing rubber band ligation with excisional haemorrhoidectomy. Br J Surg 2005;92:1481-7.

20 Jorge JM, Wexner SD. Etiology and management of fecal incontinence. Dis Colon Rectum 1993;36:77-97.

21 Criteria of diagnosis and therapeutic effect of disease and syndromes in traditional Chinese medicine, 1994. Available: http:// www.cnki.com.cn/Article/CJFDTotal-HBZZ200202046.htm

22 Katdare MV, Ricciardi R. Anal stenosis. Surg Clin North Am 2010;90:137-45. Table of Contents.

23 Balestroni G, Bertolotti G. [EuroQol-5D (EQ-5D): an instrument for measuring quality of life]. Monaldi Arch Chest Dis 2012;78:155-9.

24 EuroQol Group. EuroQol - a new facility for the measurement of health-related quality of life. Health Policy 1990;16:199-208.

25 Nyström P-O, Qvist N, Raahave D, et al. Randomized clinical trial of symptom control after stapled anopexy or diathermy excision for haemorrhoid prolapse. Br J Surg 2010;97:167-76.

26 Lu M, Yang B, Liu Y, et al. Procedure for prolapse and hemorrhoids vs traditional surgery for outlet obstructive constipation. World $\mathrm{J}$ Gastroenterol 2015;21:8178-83.

27 Bhatti MI, Sajid MS, Baig MK. Milligan-Morgan (open) versus Ferguson haemorrhoidectomy (closed): a systematic review and meta-analysis of published randomized, controlled trials. World $\mathrm{J}$ Surg 2016;40:1509-19.

28 Majumder KR, Karmakar R. Short term and long term outcome following stapled haemorrhoidopexy. Mymensingh Med $\mathrm{J}$ 2019;28:866-71.

29 Emile SH, Elfeki H, Sakr A, et al. Transanal hemorrhoidal dearterialization (THD) versus stapled hemorrhoidopexy (SH) in treatment of internal hemorrhoids: a systematic review and meta-analysis of randomized clinical trials. Int $J$ Colorectal Dis 2019;34:1-11. 